Soft Dentin Results in Unique Flexible Teeth in Scraping Catfishes

Author(s): Tom Geerinckx, Ann Huysseune, Matthieu Boone, Myriam Claeys, Marjolein

Couvreur, Barbara De Kegel, Peter Mast, Luc Van Hoorebeke, Kim Verbeken and Dominique

Adriaens

Reviewed work(s):

Source: Physiological and Biochemical Zoology, (-Not available-), p. 000

Published by: The University of Chicago Press

Stable URL: http://www.jstor.org/stable/10.1086/667532

Accessed: 06/08/2012 06:54

Your use of the JSTOR archive indicates your acceptance of the Terms \& Conditions of Use, available at

http://www.jstor.org/page/info/about/policies/terms.jsp

JSTOR is a not-for-profit service that helps scholars, researchers, and students discover, use, and build upon a wide range of content in a trusted digital archive. We use information technology and tools to increase productivity and facilitate new forms of scholarship. For more information about JSTOR, please contact support@jstor.org. 


\section{Soft Dentin Results in Unique Flexible Teeth in Scraping Catfishes}

\author{
Tom Geerinckx ${ }^{1, *}$ \\ Ann Huysseune ${ }^{2}$ \\ Matthieu Boone ${ }^{3}$ \\ Myriam Claeys ${ }^{4}$ \\ Marjolein Couvreur ${ }^{4}$ \\ Barbara De Kegel ${ }^{1}$ \\ Peter Mast ${ }^{5}$ \\ Luc Van Hoorebeke ${ }^{3}$ \\ Kim Verbeken ${ }^{5}$ \\ Dominique Adriaens ${ }^{1}$ \\ ${ }^{1}$ Evolutionary Morphology of Vertebrates, Ghent University, \\ K. L. Ledeganckstraat 35, 9000 Ghent, Belgium; \\ ${ }^{2}$ Evolutionary Developmental Biology, Ghent University, \\ K. L. Ledeganckstraat 35, 9000 Ghent, Belgium; ${ }^{3}$ Ghent \\ University Centre for Tomography (UGCT), Department of \\ Physics and Astronomy, Ghent University, Proeftuinstraat 86, \\ 9000 Ghent, Belgium; ${ }^{4}$ Nematology Unit, Department of \\ Biology, Ghent University, K. L. Ledeganckstraat 35, 9000 \\ Ghent, Belgium; ${ }^{5}$ Department of Materials Science and \\ Engineering, Ghent University, Technologiepark 903, 9052 \\ Zwijnaarde, Belgium
}

Accepted 6/23/2012; Electronically Published 8/3/2012

\begin{abstract}
Teeth are generally used for actions in which they experience mainly compressive forces acting toward the base. The ordered tooth enamel(oid) and dentin structures contribute to the high compressive strength but also to the minor shear and tensile strengths. Some vertebrates, however, use their teeth for scraping, with teeth experiencing forces directed mostly normal to their long axis. Some scraping suckermouth catfishes (Loricariidae) even appear to have flexible teeth, which have not been found in any other vertebrate taxon. Considering the mineralized nature of tooth tissues, the notion of flexible teeth seems paradoxical. We studied teeth of five species, testing and measuring tooth flexibility, and investigating tooth (micro)structure using transmission electron microscopy, staining, computed tomography scanning, and scanning electron microscopy-energy-dispersive spectrometry. We quantified the extreme bending capacity of single teeth (up to $180^{\circ}$ ) and show that reorganizations of the tooth (micro)structure and extreme hypomineralization of the dentin are adaptations preventing breaking by allowing flexibility.
\end{abstract}

\footnotetext{
* Corresponding author; e-mail: tom.geerinckx@ugent.be.
}

Physiological and Biochemical Zoology 85(5):000-000. 2012. (C) 2012 by The University of Chicago. All rights reserved. 1522-2152/2012/8505-2006\$15.00. DOI: $10.1086 / 667532$
Tooth shape and internal structure appear to be optimized for bending in one direction, which is expected to occur frequently when feeding (scraping) under natural conditions. Not all loricariid catfishes possess flexible teeth, with the trait potentially having evolved more than once. Flexible teeth surely rank among the most extreme evolutionary novelties in known mineralized biological materials and might yield a better understanding of the processes of dentin formation and (hypo)mineralization in vertebrates, including humans.

\section{Introduction}

The teeth of vertebrate animals are highly variably shaped; they are used for biting, grasping, crushing, cutting, and chewing. These functions are derivatives of the same basic action, in which teeth experience mainly compressive forces acting mostly downward, toward the base of the tooth. Teeth are wonderfully adapted for this, as they contain hard, mineralized tissuesdentin, enamel, and enameloid-including the hardest biological materials nature has produced (Lund et al. 1992; Currey 1999). The ordered microstructure of the hydroxyapatite crystals and the organic components of the enamel(oid) and the dentin contributes to the tooth strength by adding the minor but necessary degree of tensile and shear strength, directing forces and preventing cracks (Waters 1980; Watanabe et al. 1996; Marshall et al. 1997; Currey 1999; Kinney et al. 2001; Mannocci et al. 2004; Miguez et al. 2004).

Few living animals use their teeth for trophic functions that are not related to biting. Two trophic functions exist that cause food or substrate reaction forces to act predominantly normal to the long axis of teeth: filtering and scraping. In view of these functions, the advantages of teeth being hard and stiff, withstanding compression with a high mineral content, imply the drawback of being very brittle and, hence, susceptible to fracture. A certain degree of bending capacity would be favorable for such teeth. Mechanically, a bending tooth would experience a great amount of tension, which sounds rather counterintuitive for teeth in general. Dental tissues seem not well suited for flexibility.

Evolutionarily, teeth have been lost and functionally replaced by more flexible keratinous materials that allow feeding by filtering (e.g., in baleen whales) or scraping (e.g., in tadpoles and several cypriniform fishes; Arens 1994). The long and slender teeth of the fossil pterosaur Pterodaustro guiñazui are thought to have functioned as filtering bristles, much like the keratinous flamingo bristles (Chiappe and Chinsamy 1996). Given their very high length-width ratio these genuine teeth presumably could very well bend to some degree without break- 


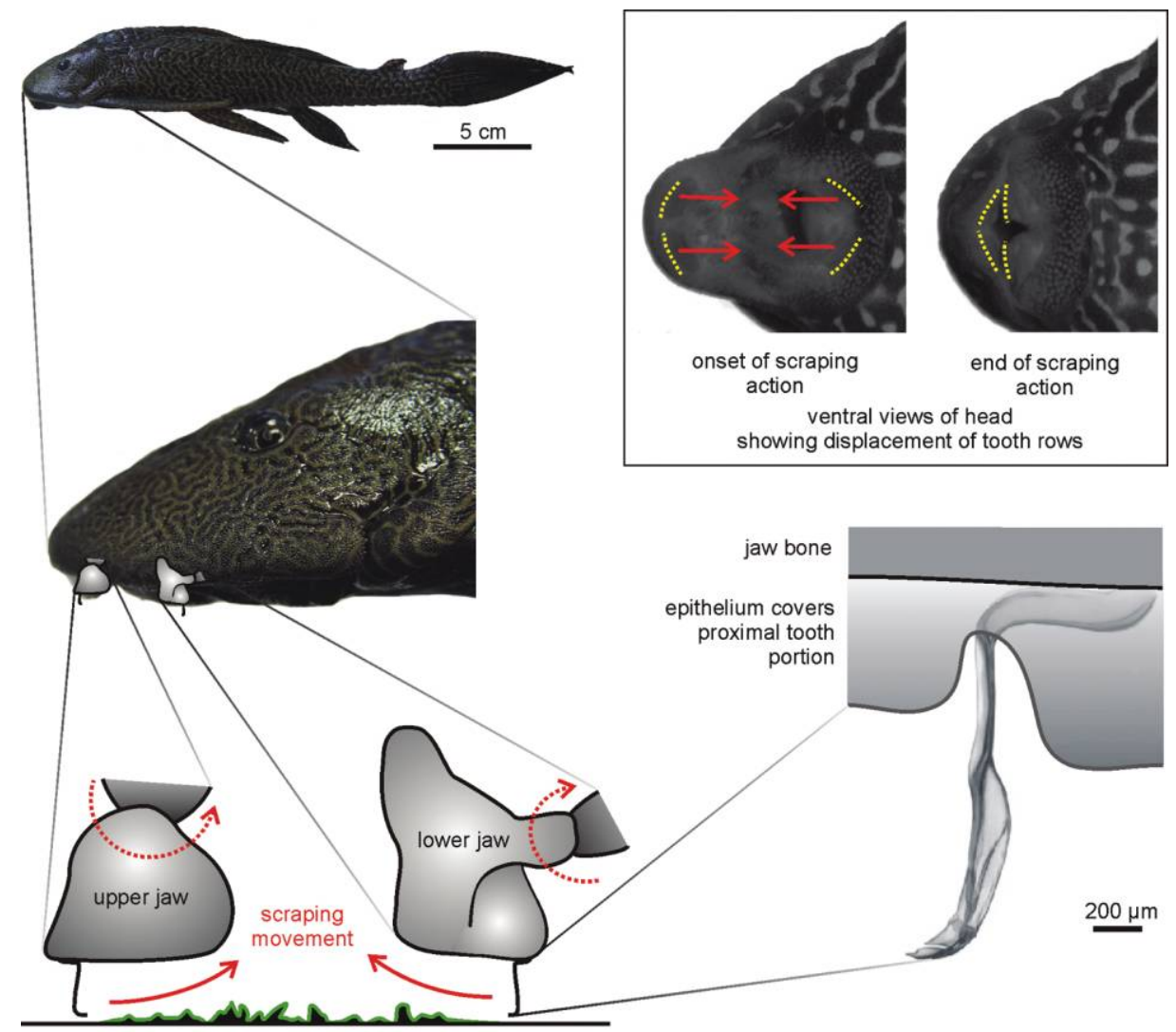

rough substrate with food

Figure 1. Diagram showing jaw and tooth position in Pterygoplichthys disjunctivus. Left and right upper and lower jaws each bear one row of teeth that are scraped along the substrate during jaw rotation (arrows). The inset shows the extent of the movement of the tooth rows (dotted lines) in one scraping motion.

ing (Currey 1999); no information is available on the mineralization degree of P. guiñazui teeth. Few living taxa have evolved truly scraping teeth. These then are often bristle-like and/or strongly curved, with a gouge-like tip. Examples include the surgeonfishes (Acanthuridae), butterflyfishes (Chaetodontidae), combtooth blennies (Blenniidae), and other marine reefgrazing taxa (Motta 1984; Purcell and Bellwood 1993; Christiansen et al. 2010), and the freshwater suckermouth catfishes of the families Mochokidae, Astroblepidae, and Loricariidae. This list is certainly not exhaustive, and quite little is known about the tooth properties of most grazing or scraping fishes. Mobile tooth joints have been reported (e.g., Fink 1981; Motta 1984), indicating the apparent benefit of teeth that are not totally rigid or immobile relative to the jaw. Still, flexibility in tooth material itself is hitherto unknown.

Loricariidae are by far the world's largest catfish family, with over 700 known species (Ferraris 2007), all scraping their food, although some have reduced teeth and might merely suck food particles. Teeth of loricariids range from slim to stout depending on the species. Diets can include algae, detritus, invertebrates, and plant material, grazed off the substrate by elaborate scraping jaw movements thanks to highly mobile, ventrally directed upper and lower jaws (fig. 1; Adriaens et al. 2009). Some loricariid species are known to scrape off bits of wood; they have stout, rigid teeth (e.g., Panaque nigrolineatus; Nelson 1999; Geerinckx et al. 2007). In several other species of different lineages within the loricariid family, scraping algae or detritus, it was noticed that the gracile teeth have a flexible middle portion, apparently reducing tooth fracture during scraping by somehow bending (Geerinckx et al. 2007).

Given the paradoxical notion of truly flexible tooth tissue, the aim of this study was to characterize the tooth structure and quantify the tooth bending capacity. We tested the hypothesis that the organization of the loricariid tooth (micro)structure displays adaptations to prevent fracture by reducing brittleness, and thus allowing flexibility. We propose that these characters match the peculiar feeding ecology of these animals and therefore may have boosted loricariid cladogenesis.

\section{Material and Methods}

\section{Material}

Adult specimens of five different commercially obtainable loricariid species were chosen for this study: Sturisoma aureum (Steindachner 1900) and Rineloricaria parva (Boulenger 1895; both subfamily Loricariinae), and Ancistrus triradiatus Eigen- 

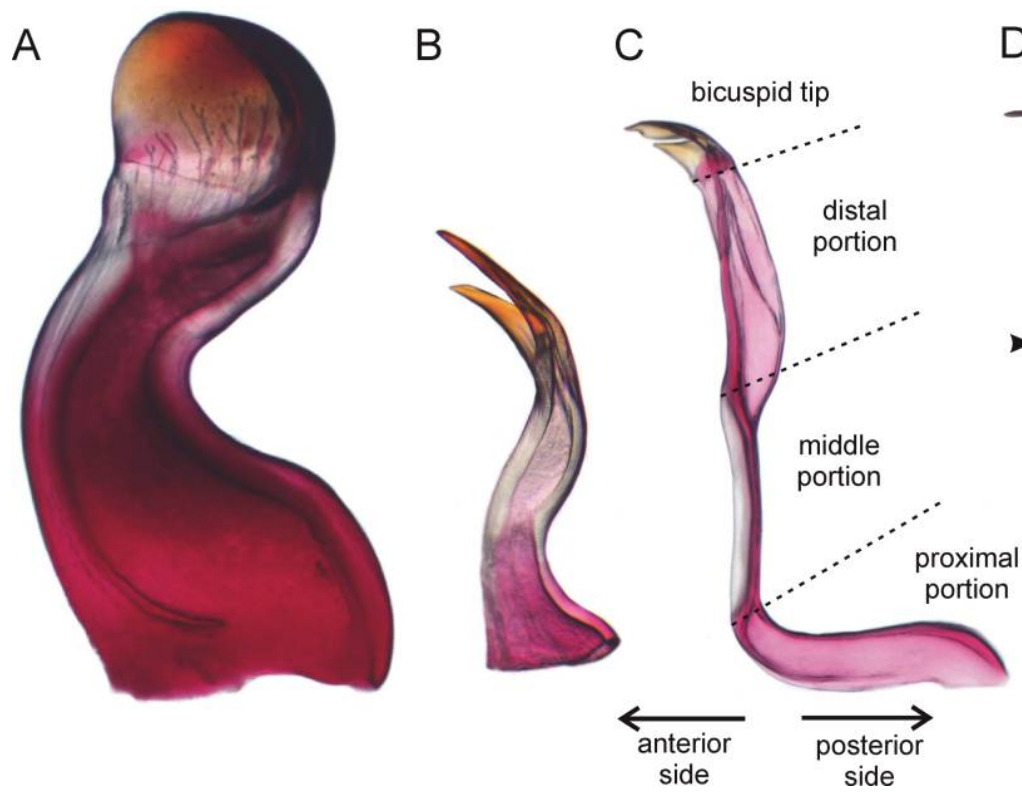

D

$\mathrm{E}$

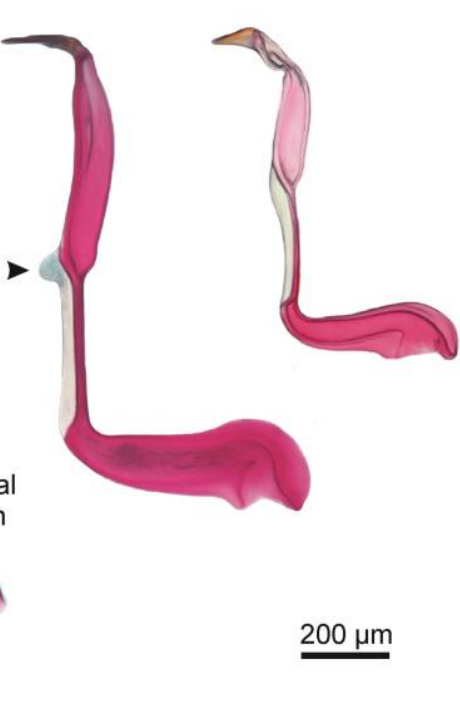

Figure 2. Alizarin- and alcian-stained teeth of Panaque nigrolineatus (A), Rineloricaria parva (B), Pterygoplichthys disjunctivus (C), Ancistrus triradiatus $(D)$, and Sturisoma aureum $(E)$. Teeth of the latter three species have a middle portion that differs from the other tooth parts by an anterior zone that is not stained by alizarin. Teeth of $A$. triradiatus have an additional unmineralized anterior bulge (arrowhead). Teeth are bicuspid except in P. nigrolineatus.

mann 1918, Pterygoplichthys disjunctivus (Weber 1991), and $\mathrm{Pa}$ naque nigrolineatus (Peters 1877) (all subfamily Hypostomatinae).

\section{Fixation and Staining}

Whole teeth were extracted from the lower jaw of specimens that had been sacrificed using an overdose of the anesthetic MS222 benzocaine (Sigma Chemical). Teeth were then fixed in a $10 \%$ buffered formalin solution or a paraformaldehyde-glutaraldehyde mixture $(1.5 \%$ each, cacodylate buffered at $\mathrm{pH} 7.4$; for scanning and transmission electron microscopy [SEM and TEM, respectively] analyses). According to the Belgian law of August 14,1986 , on animal welfare and to the policy of Ghent University, no specific ethical approval is needed when fish specimens necessary for research are killed in the least painful way. Several teeth of all five species were stained with alizarin for calcified material $(0.1 \%$ alizarin in distilled water, with a $0.5 \% \mathrm{KOH}$ solution added until stain turns purple); some teeth of each species were first stained with alcian blue for mucopolysaccharides and glycosaminoglycans ( $1 \mathrm{mg}$ in $7 \mathrm{~mL}$ ethanol and $3 \mathrm{~mL}$ glacial acetic acid). One specimen per species was sacrificed for dissection of the jaws, in order to clarify the tooth replacement pattern (number and positions of replacement teeth).

\section{Micro-Computed Tomography (CT) Scanning}

Two teeth of $A$. triradiatus and one of $R$. parva were scanned using one of the modular micro-CT setups of the Ghent University Centre for X-ray Tomography (UGCT), visualizing hard versus softer parts of the teeth. The x-ray setup consisted of a FeinFocus FXE160.51 transmission tube head at 60-kV tube voltage and a Photonic Science VHR detector with a GadOx scintillator. For each scan, 800 projection images of $967 \times 644$ pixels were recorded, covering $360^{\circ}$, with a total exposure time per projection of $10 \mathrm{~s}$. The raw data were processed and reconstructed using the CT software Octopus (developed in-house; Vlassenbroeck et al. 2007) at an isotropic voxel size of $(1,300 \mathrm{~nm})^{3}$. An

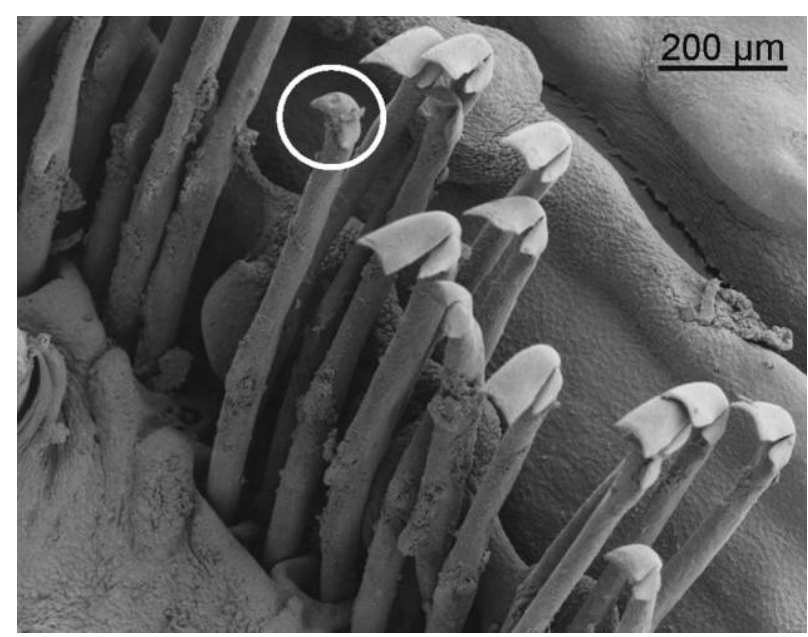

Figure 3. Scanning electron micrograph showing the wear of the tooth cusps in Ancistrus triradiatus (example of completely worn cusp in circle). Notice the loose embedding in the soft dental epithelium and the ridge at the posterior side of the teeth (right side of picture). 
A

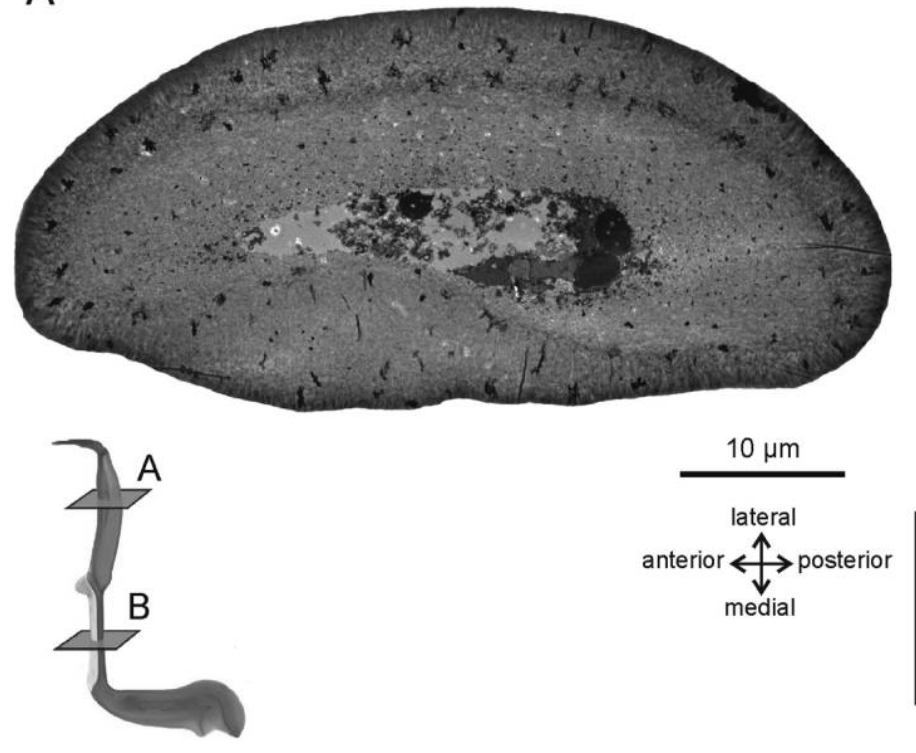

B

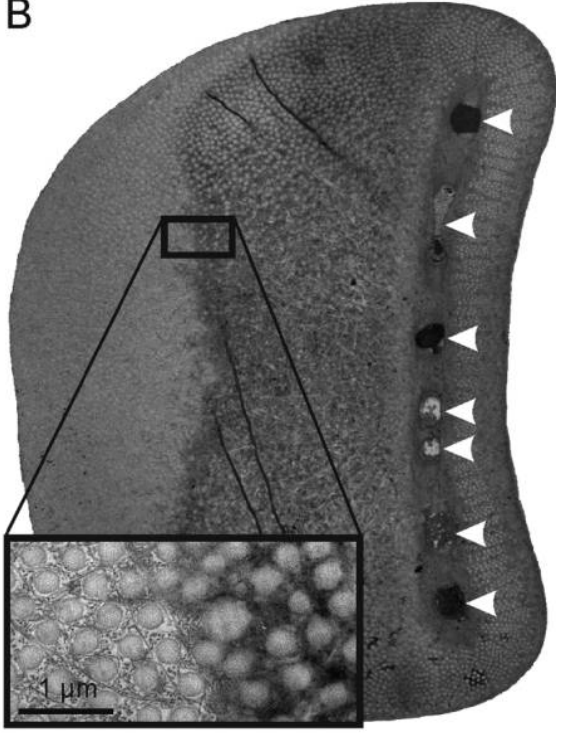

Figure 4. Transmission electron micrographs of transverse sections through the distal $(A)$ and the middle $(B)$ portions of a tooth of Ancistrus triradiatus. $B$, Pulp cavity is subdivided into several canals lying at the posterior side of the tooth (arrowheads). Inset shows nearly unmineralized (left) and apparently more mineralized (right) dentin. Cross sections of thick longitudinal collagen fibers are easily visible in $B$; much narrower collagen fibers run in all directions. A color version of this figure is available in the online edition of Physiological and Biochemical Zoology.

additional $A$. triradiatus tooth was scanned at higher resolution (FeinFocus FXE160.51 at $80 \mathrm{kV}$ and with a Varian PaxScan 2520 aSi flat panel with CsI scintillator). With 1,000 projection images of $1,880 \times 1,496$ pixels per scan (5.4 s exposure time per projection), the data were reconstructed at an isotropic voxel size of (516 nm) $)^{3}$. VGStudio Max 2.0 (Volume Graphics, Heidelberg) was used for three-dimensional rendering.

\section{SEM and Energy-Dispersive X-Ray Spectroscopy (EDS)}

SEM pictures were taken of fixed, dried, and gold-coated jaws, teeth, and internal tooth tissues of all species, using a Balzers SCD 040 gold sputter coater and a Jeol JSM-840 microscope operating at $15 \mathrm{kV}$; magnification up to $\times 25,000)$. For EDS, individual teeth were manually cut transversely or longitudinally, and the resulting samples were gold coated using a BalTec SCD 005 gold sputter coater. The material was then examined using a Philips XL30 ESEM (15 kV; magnification up to $\times 9,600)$. SEMEDS was performed with an EDAX EDS system. Element compositions were measured in various portions of the cut teeth as averages of areas of $20-150 \mu \mathrm{m}^{2}$. Weight percentages were calculated after ZAF correction for the effects of atomic number, absorption, and fluorescence.

\section{TEM}

Both transverse and longitudinal sections of teeth of an $A$. triradiatus specimen were used for a TEM examination of the structure of tooth tissues. Ice-cooled Karnovsky fixation (2\% paraformaldehyde, $2.5 \%$ glutaraldehyde in $0.2 \mathrm{M}$ sodium cac- odylate buffer, $\mathrm{pH}$ 7.4) in a partial vacuum over $24 \mathrm{~h}$ was followed by further fixation with EDTA added to the fixative for decalcification $(12 \mathrm{~h})$. Still at ca. $4^{\circ} \mathrm{C}$, teeth were put in cacodylate buffer $0.1 \mathrm{M}(24 \mathrm{~h}$, one change) and then in reduced osmium for postfixation $(16 \mathrm{~h})$. Washing for $10 \mathrm{~min}$ in cacodylate buffer was followed by an ethanol dehydration series. Over $5 \mathrm{~d}$, teeth were impregnated in a 50/50 propylene oxide/ epon mixture. After overnight propylene oxide evaporation and a change into fresh epon for $5 \mathrm{~h}$, final embedding was done at $60^{\circ} \mathrm{C}$ over $24 \mathrm{~h}$. Various regions of teeth were cut transversely or longitudinally at 70-80-nm thickness, using a Reichert Ultracut S ultramicrotome (Leica, Vienna) with a diamond knife (Diatome, Biel, Switzerland), mounted on Formvar-coated single-slot copper grids (Agar Scientific, Stansted, UK) and contrast stained with uranyl acetate and lead citrate (EM stain; Leica). Electron microscopy was performed using a Jeol JEM 1010 (Jeol, Tokyo) operating at $60 \mathrm{kV}$. Images were digitized using a DITABIS system (Pforzheim, Germany).

\section{Bending Experiments}

Ten or more freshly extracted, unfixed teeth of A. triradiatus, $P$. disjunctivus, and $S$. aureum were put on a glass slide in an aqueous medium, glued at their base, and two teeth per species were given some minute markings with a fine alcohol marker pen. With a fine needle, teeth were manually bent anteriorly (forward) and posteriorly (backward, as during scraping), and with an Olympus SZX9 stereoscopic microscope and a mounted Colorview 8 digital camera, 10 pictures were taken per tooth, in upright position and in varying degrees of anterior or pos- 

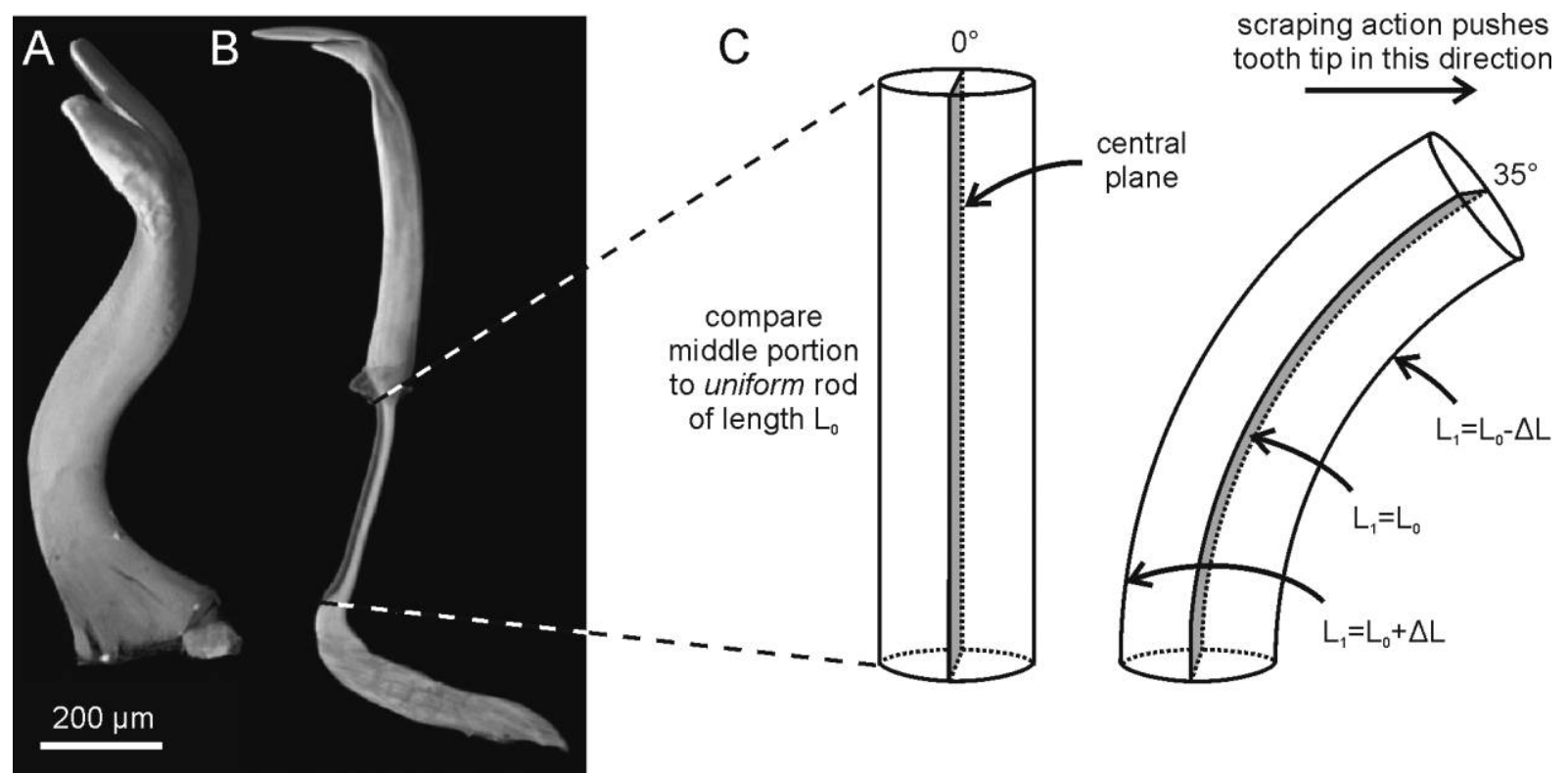

Figure 5. Computed tomography scan images of teeth of Rineloricaria parva $(A)$ and Ancistrus triradiatus (B), illustrating the hypomineralized anterior zone in the middle portion of $A$. triradiatus tooth only (partially shrunken because of dehydration). $C$, If the middle portion was a flexible rod with uniform material properties, a posterior bending would cause any axis on the central plane to be neutral (i.e., without length change: $L_{1}=L_{0}$ ). A more anterior axis would elongate by a distance $\Delta L$, while a more posterior axis would shorten by a distance $\Delta L$. The $\Delta L$ is dependent on distance from the neutral axis and degree of bending. An anterior bending would have opposite effects.

terior bending. Using AnalySIS 5.0 software (Olympus SIS, Munster, Germany), measurements were taken of five longitudinal axes drawn on the middle tooth portion (in lateral view), using the markings as an aid to ensure that axes were drawn and measured on exactly the same spots. The lengths of these five axes, all changing in the 10 pictures per tooth (decreasing or increasing), provide information on the location of compression and tension during flexion. AnalySIS 5.0 was also used for acquiring some morphometric data of 12 fresh teeth per species.

In mechanics, bending (or flexure) characterizes the behavior of a slender element (e.g., a thin tooth) subjected to an external load applied normal to the longitudinal axis of the element (on or near the tip, as during scraping). Bending capacity then is the capacity of the tooth to bend to a certain angle without breaking. Shear stresses will be (unequally) present throughout the tooth. But bending stresses also include tensile stress on one side of the tooth, and compressive stress on the other side. Bending occurs only when the material can deform: the former side (tensile stress) will lengthen, and the latter side (compressive stress) will shorten. Axes drawn on the 10 pictures can visualize this. A neutral axis will be present where the material will not change length. Naturally, material properties (e.g., brittleness of crystals) will strongly affect the local bending capacity of a tooth.

The experiment was repeated with eight or more additional teeth per species, without markings. Teeth of $P$. nigrolineatus and $R$. parva could not be bent without immediate breaking, so the experiment could not be fully performed on these.

\section{Results}

\section{Tooth Structure}

The choice of species allows to compare flexible versus rigid teeth in the two largest loricariid subfamilies: flexible (Sturisoma aureum) versus rigid (Rineloricaria parva) in Loricariinae and flexible (Ancistrus triradiatus and Pterygoplichthys disjunctivus) versus rigid (Panaque nigrolineatus) in Hypostomatinae (fig. 2). For ease of interpretation of tooth parts, we describe four portions in flexible loricariid teeth (fig. 2C): (1) the proximal (basal) portion is thick and is covered by the epithelium; (2) the middle portion, forming a $90^{\circ}$ angle with the former portion and standing upright from the epithelium, is the thinnest in lateral view and ends with an anterior bulge in A. triradiatus; (3) the following, distal portion then bears (4) the curved bicuspid tip. The different portions are less pronounced in the nonflexible teeth of $P$. nigrolineatus and $R$. parva. Teeth of $P$. nigrolineatus are unicuspid. In the local frame of reference of each tooth, we define the side with the cusp(s) as the "anterior" side (fig. 2C).

TEM investigation shows that the teeth of all five species are composed mostly of dentin, with enameloid dominating only the far distalmost portion (the tip). The proximal tooth portion of all species is embedded in the epithelium (figs. 1, 3). The stout (inflexible) teeth of $P$. nigrolineatus are well anchored to the jaw (less so in $R$. parva). Teeth of A. triradiatus, $P$. disjunctivus, and S. aureum are movably attached to the jaw.

In the middle portion of teeth of these latter three species, the pulp cavity narrows and is divided into 6-10 narrow canals, 
A

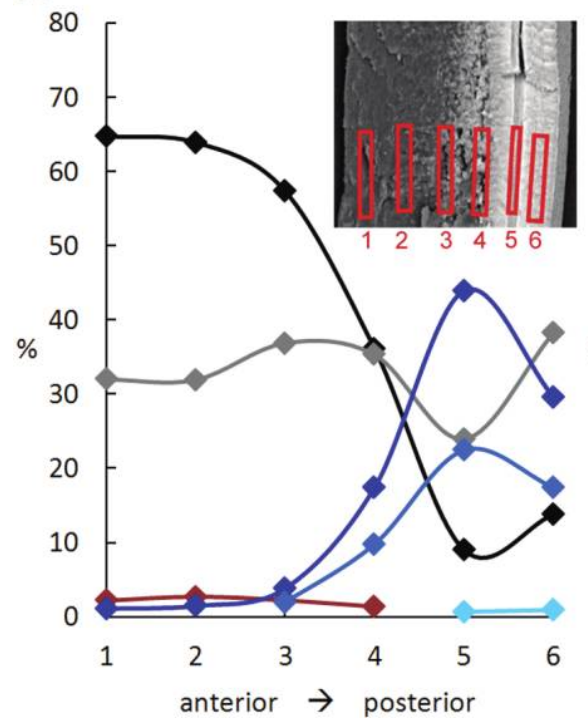

B

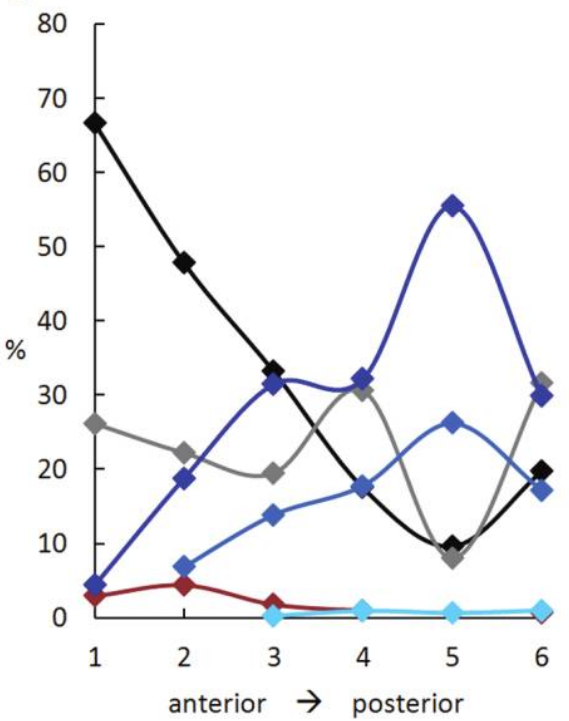

C

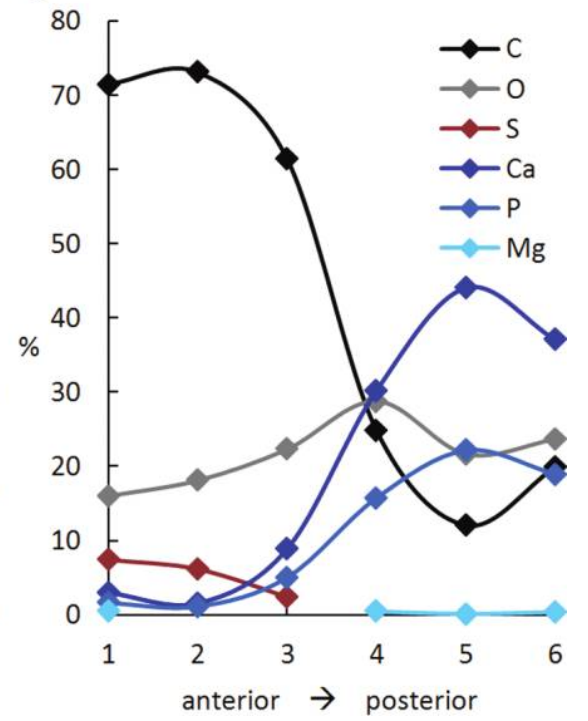

Figure 6. Element composition in the flexible tooth portion. Graphs show the anteroposterior gradient in element composition (weight percentages) on a horizontal section halfway through the middle portion of teeth in Ancistrus triradiatus (A), Pterygoplichthys disjunctivus (B), and Sturisoma aureum $(C)$. Values are based on six $20-150-\mu \mathrm{m}^{2}$ areas from anterior (1) to posterior (6) on a longitudinal section, as shown for A. triradiatus (inset). Note the calcification peak at point 5, representing dentin between the pulp cavity canals. $\mathrm{C}=\mathrm{carbon}, \mathrm{O}=$ oxygen, $\mathrm{S}=$ sulphur, $\mathrm{Ca}=$ calcium, $\mathrm{P}=$ phosphorus, $\mathrm{Mg}=$ magnesium.

positioned eccentrically toward the posterior side of the tooth (fig. 4). TEM imaging shows that in the flexible teeth these canals are surrounded and separated by a thin layer of matrix with little visible collagen. Numerous collagen fibers of various sizes (inset, fig. 4) are visible in all other parts of the tooth. Some degrees of canal-like subdivision of the pulp cavity are present at its distal end (visible on fig. $2 A$ ).

Also in this middle portion, an anterior zone is defined by the lack of alizarin staining, strongly suggesting that it lacks calcification (fig. 2), and by CT scanning, suggesting that it contains less-dense material (fig. 5B). Strikingly, the tissue of this anterior zone of the middle portion slightly and reversibly deforms even when gently touching it with a needle. Using EDS, the element composition in the middle portion of $A$. triradiatus, $P$. disjunctivus and $S$. aureum teeth proved to grade from a strongly hypomineralized anterior (e.g., 1\%-2\% Ca) to a more mineralized posterior region (ca. 29\%-35\% Ca), with the region immediately around the posteriorly located pulp canals being most extensively mineralized (up to $55 \% \mathrm{Ca}$; fig. 6). As a reference to these numbers, Arnold and Gaengler (2007) found a Ca weight percentage of about 36.6\% $\pm 7.2 \%$ in human dentin. In the nonbendable parts of the tooth (proximal and distal portions), the microstructure (fig. 4) and the element composition of the dentin are generally most similar to the middle portion zone posterior to the pulp canals (posteriormost values in fig. 6; data not shown).

Tubules, common in dentinal tissues, are virtually absent in the flexible teeth (of A. triradiatus, $P$. disjunctivus, and $S$. aureum), whereas short tubules were found to be more numerous along the entirety of the rigid teeth of $P$. nigrolineatus (no clear indication in R. parva).

In A. triradiatus, an anterior bulge at the distal end of the middle portion contains an almost unmineralized matrix (e.g., $0.4 \% \mathrm{Ca}, 71 \% \mathrm{C}$ ), that is stained by alcian blue (arrowhead on fig. 2D). In the rigid teeth of $P$. nigrolineatus and $R$. parva, virtually no difference was found between the element composition at various locations in the dentin; no indications of hypomineralization were found. As mentioned above, there is no clear morphological differentiation of the S-shaped teeth of these two species into different portions.

\section{Bending Capacity}

Individual tooth bending capacity was tested after tooth extraction. It was not possible to directly observe in vivo tooth bending during feeding due to small tooth size and scraping speed. Experimental bending could be observed by using a needle to move the tooth tip while the base of the proximal portion was fixed to a glass plate on which the tooth was lying on its lateral side. In the experiments, teeth experienced forces largely normal to their long axis; also, in natural conditions the force angle will vary somewhat, as jaws are rotated, not merely translated, over the substrate (fig. 1). Attempting to bend the teeth of $R$. parva and P. nigrolineatus caused breaking without any observable bending, whereas teeth of $A$. triradiatus, $P$. disjunctivus and $S$. aureum could be bent both anteriorly and posteriorly. Tooth bending appeared to occur only in the thin, middle portion. When the other portions were specifically 

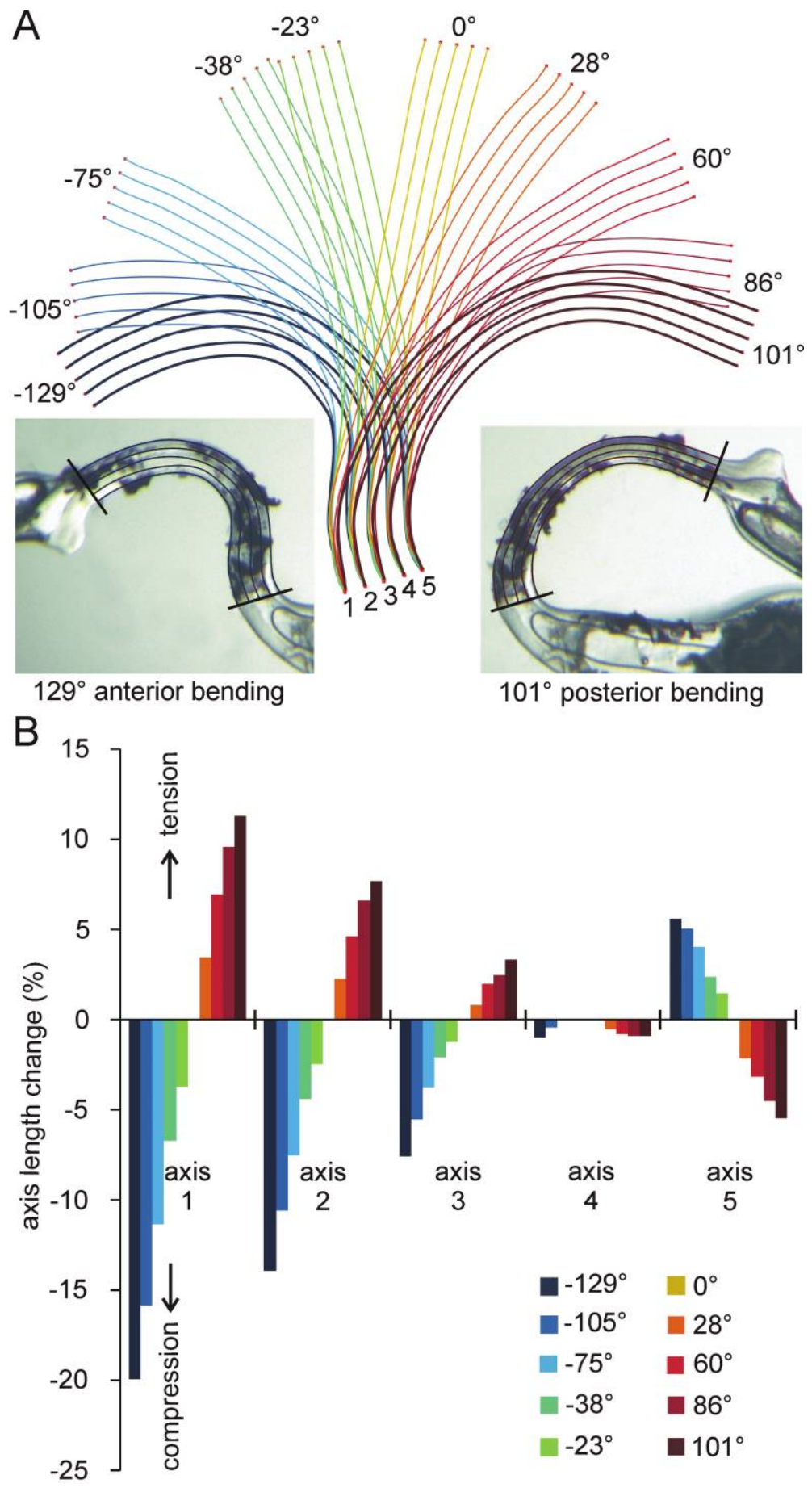

Figure 7. Bending experiments. A, Composite figure of five longitudinal axes drawn on the flexible middle portion of an Ancistrus triradiatus tooth, on 10 lateral pictures of tooth bending from $-129^{\circ}$ (anterior bending) to $101^{\circ}$ (posterior bending), with $0^{\circ}$ representing the rest position. Axis 4 lies closest to the pulp cavity and the most mineralized dentin surrounding it. Insets show the black markings that aided correct measurements of the axes on all pictures. $B$, Graph showing the length changes of the five axes. On the $Y$-axis, positive values represent material elongation; negative values represent material compression along a certain axis. Axis 4 never elongates (see text).

forced to bend (by using a needle or fine forceps) the material failed almost immediately, with some very limited sideward (lateral) bending possible in the distal portion.

A material that is bent, like the middle tooth portion, will deform differently along the inside and outside of the resulting curvature. One side will experience tensile stress and so will lengthen, while the other, compressed side shortens. In structures with uniform material properties, a neutral plane will be 
present centrally (fig. $5 C$ ). To quantify this deformation, the shortening and lengthening of five axes drawn along the length of the middle portion at different positions from anterior to posterior were measured during bending in both the anterior and the posterior directions (fig. $7 \mathrm{~A}$ ). Results consistently show that major length changes occur in the hypomineralized anterior portion and that least change is found at the level of the pulp cavity canals (surrounded by the most mineralized dentin, see above; axis 4 on fig. 7). Interestingly, this "most mineralized" region slightly shortens in both the forward and the backward bending in each test (fig. $7 B$ ).

Experimentally, teeth of A. triradiatus could be bent farthest, and more repeatedly, without breaking. Backward bending was spatially constrained by the distal tooth side bumping against the tooth base (at about $100^{\circ}$; see right inset on fig. $7 \mathrm{~A}$ ), whereas forward bending could reach its spatially constrained maximum at about $180^{\circ}$. In all three flexible-toothed species, breaking was always initiated during forward bending, somewhere at the stretched posterior, more mineralized side. Fractures are typically normal to the long axis of the tooth and seldom proceed into the hypomineralized anterior half of the tooth. Among the three flexible-toothed species, the dimensions of the middle tooth portion differ, with $A$. triradiatus having the narrowest and $P$. disjunctivus the broadest middle portion (width/length ratio $[ \pm \mathrm{SD}]$ of $10.65 \% \pm 0.66 \%$ and $19.05 \% \pm 1.68 \%$, respectively).

\section{Discussion}

Combined results of local material properties and bending loadings indicate that the dentin tissues of Ancistrus triradiatus, Pterygoplichthys disjunctivus, and Sturisoma aureum are well suited to the types of loading they are most subjected to during the acts of scraping. When interpreting the performance results of the teeth of these species, it is important to realize that in vivo, the teeth are not experiencing such extremely high degrees of bending, although the exact natural maxima are not known. Their placement in the oral epithelium (figs. $1 D, 3$ ) dampens bending in any direction, and the higher epithelial tissue ridge at the labial side prevents posterior bending over ca. $40^{\circ}$ in the three species examined. Teeth can be expected to be naturally subjected to posterior bending during the act of scraping (upper and lower jaw "closing"). Anterior bending might occur during repositioning of the jaws, as teeth may be dragged back over the substrate. When experiencing these "natural" degrees of in vivo flexion, teeth apparently rarely break, despite highly repetitive bending during the countless scraping movements; broken tooth shafts were very seldom observed in examined specimens. Although aquarium circumstances (plants, wood, lots of rock to feed on) were not necessarily comparable to the natural habitat, tooth fracture was nearly nonexistent. Erupted teeth, however, did show varying degrees of cusp wear, including teeth lacking part or whole of the scraping tips (fig. 3). Likely, replacement teeth erupt when older teeth with completely worn cusps are shed. In fishes, tooth families are defined as series of developmentally successive teeth taking the same place when active, each replacing the previous when that is worn and shed. In loricariid catfishes, tooth families possess up to five replacement teeth on apparently permanent dental laminae, with the highest number of replacement teeth present in species with more slender teeth (Geerinckx et al. 2007). This unusually high number likely is an adaptation to the short life span of teeth and to the consequently necessarily high turnover rate of teeth.

Increasing slenderness and flexibility of the teeth apparently correlates with the decreasing strength of tooth-to-jaw attachment, from rigid in Panaque nigrolineatus to very loose in the flexible-toothed species. As opposed to tooth flexibility, movable (vs. rigid) tooth-to-jaw attachment is known from other fish taxa as well (Fink 1981; Motta 1984; Huysseune and Sire 1998). Both tooth and tooth attachment flexibility actually serve the same purpose: providing some degree of tooth fracture resistance by decreasing the rigidity of the jaw-teeth apparatus. Attachment flexibility (by fibrous connections) has been shown to play an important role in biting in sharks and rays, which display a nice analogy to loricariid catfishes regarding tooth turnover rate and replacement pattern (e.g., Ramsay and Wilga 2007; Dean et al. 2008).

Dentin is typically well mineralized and rather brittle, appearing to have been optimized to deal with compression, not tension; compressive strength of dentin is always found to be much higher than tensile strength (Waters 1980). In human dentin, fracture has been observed to be strain based (Nalla et al. 2003). It appears that in loricariid catfishes, an evolutionary tendency has arisen that improves the strain-resisting capacities of dentin by reducing the mineral content in the zone that experiences most tension.

However, even for rather well-mineralized biomaterials, the brittleness of the mineral constituent can become negligible, allowing for substantial flexibility, when at least one of the following conditions applies: very small crystal size (Gao et al. 2003; Ji and Gao 2004) or teeth with a very small width/length ratio. At least the latter is thought to have enabled the long slender teeth of the pterosaur Pterodaustro guiñazui to act as somewhat flexible bristles (Chiappe and Chinsamy 1996; Currey 1999). In flexible loricariid teeth, the presence of only a very thin zone of truly highly mineralized dentin, positioned eccentrically toward the posterior, would then in a similar way strongly decrease the chance of this brittle dentin breaking during scraping: scraping, and thus posterior bending, causes tension at the anterior side. The neutral axis of the tooth (the length of which does not change during bending) appears to be located near axis 4 in figure 7 . The fact that axis 4 somewhat shortens in both anterior and posterior bending is rather puzzling, but might suggest a shift in the neutral axis position between both bending directions. Knowledge of the dentin nanostructure along the middle portion may shed light on this interesting property.

In the flexible-toothed loricariid catfishes, it is not clear what has caused the pulp cavity to be located eccentrically toward the posterior. The subdividing of the pulp cavity into several canals might be related to a strengthening of this region, pre- 


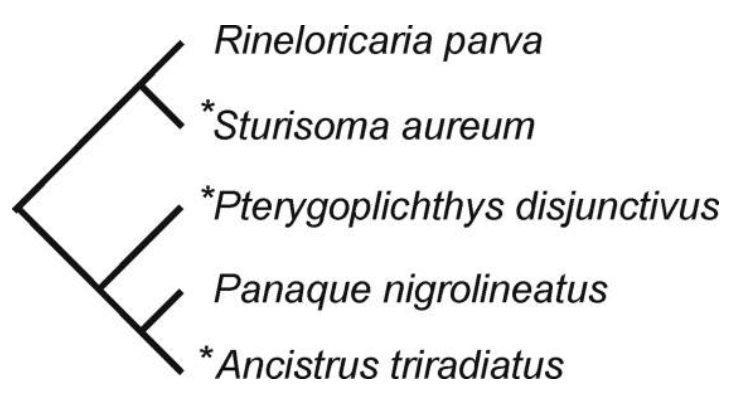

Figure 8. Phylogenetic relationships of studied specimens following Armbruster (2004). Asterisks mark species with flexible teeth (see also fig. 2).

venting too large "weak spots." Absence of tubules in the dentin could be an adaptation for increased tensile strength. High values of tensile strength in human dentin are associated with low tubule densities (Mannocci et al. 2004). Still, in a study on tensile crack propagation on the nanoscale, no evidence was found that tubules in human dentin substantially affect the process of crack initiation and growth (Nalla et al. 2003).

The largely longitudinally directed collagen fibers, found ubiquitously in the anterior zone of the loricariid flexible teeth, which is prone to tension during scraping, are interpreted here as an adaptation for increased tensile strength and crack pre $\rightarrow$ vention (Watanabe et al. 1996). Ultimate tensile strength in human dentin has been found to be highest along the direction of collagen fibers (Miguez et al. 2004).

The sulphur predominantly found in the least calcified loricariid dentin (fig. 6) can be assumed to be part of sulphatec $\rightarrow$ glycosaminoglycans of proteoglycans, various types of which are known to be present in dentin (Hess and Lee 1952; Leblond et al. 1955; Tsuchiya et al. 2001). Sulphated proteoglycans ar $\rightarrow$ more abundant in predentin (Wiesmann et al. 1995; Zhang et al. 1995), and some are reported to be degraded when predentin is converted into dentin (Fukae et al. 1994; Lormée et al. 1996). Thus, the higher $\mathrm{S}$ content of the less mineralized dentin ir $\rightarrow$ these loricariids may reflect a controlled incomplete maturation of predentin into dentin.

The trait of flexible teeth is rather widely distributed in tha $\rightarrow$ loricariid family: it has been found in members of three subfamilies (Geerinckx et al. 2007). Inflexible teeth are present ir $\rightarrow$ at least two of these subfamilies (species $P$. nigrolineatus and Rineloricaria parva); numerous loricariid species remain to be tested for tooth properties. Given the spread of the studied species with and without flexible teeth in the loricariid phylogeny (Armbruster 2004), it is not sure whether the trait o $\rightarrow$ flexible teeth has evolved several times, or only once, with at least two subsequent losses of the trait (fig. 8).

The existence of truly hypomineralized teeth in other fish taxa is well possible, as scraping species are present in various habitats, especially coral reefs. Whether in these fishes the teeth experience significant forces normal to their long axis, is not known. Given the predominance of teeth used as compressivi $\rightarrow$ tools in other vertebrates, such hypomineralization is most probably nonexistent. While pathological forms of dentin hypomineralization are known in humans (Sreenath et al. 2003; Ye et al. 2004), they are not at all comparable to the much higher extent of hypomineralization seen in loricariids (soft loricariid dentin deforms even when gently touched). Understanding, for example, the etiology of disorders like dentinogenesis imperfecta III (Ye et al. 2004) might very well benefit from a better knowledge of the regulation and genetic background of hypomineralization in loricariids with flexible teeth versus closely related species with fully mineralized teeth. A relatively simple regulatory mechanism may lie at the basis of such a drastic counterintuitive change in material properties, from dentin suited for biting to dentin suited for bending.

\section{Acknowledgments}

We thank Wim Van Paepegem for his useful comments on bending experiment techniques. T.G. is a postdoctoral researcher of the Fund for Scientific Research in Flanders, Belgium (FWO-Vl). Research was funded by FWO grant G.0355.04.

\section{Literature Cited}

Adriaens D., T. Geerinckx, J. Vlassenbroeck, L. Van Hoorebeke, and A. Herrel. 2009. Extensive jaw mobility in suckermouth armoured catfishes (Loricariidae): a morphological and kinematic analysis of a substrate scraping feeding apparatus. Physiol Biochem Zool 82:29-39.

Arens W. 1994. Striking convergence in the mouthpart evolution of stream-living algae grazers. J Zool Syst Evol Res 32: 319-343.

Armbruster J.W. 2004. Phylogenetic relationships of the suckermouth armoured catfishes (Loricariidae) with emphasis on the Hypostominae and the Ancistrinae. Zool J Linn Soc 141: $1-80$.

Arnold W.H. and P. Gaengler. 2007. Quantitative analysis of the calcium and phosphorus content of developing and permanent human teeth. Ann Anat 189:183-190.

Chiappe L.M. and A. Chinsamy. 1996. Pterodaustro's true teeth. Nature 379:211-212.

Christiansen N.A., A. Kemp, and I.R. Tibbetts. 2010. Are the oral teeth of combtooth blennies (Salariini: Teleostei) merely combs? Env Biol Fish 87:205-216.

Currey J.D. 1999. The design of mineralized hard tissues for their mechanical functions. J Exp Biol 202:3285-3294.

Dean M.N., J.B. Ramsay, and J.T. Schaefer. 2008. Tooth reorientation affects tooth function during prey processing and tooth ontogeny in the lesser electric ray, Narcine brasiliensis. Zoology 111:123-134.

Ferraris C.J. 2007. Checklist of catfishes, recent and fossil (Osteichthyes: Siluriformes), and catalogue of siluriform primary types. Zootaxa 1418:1-628.

Fink W.L. 1981. Ontogeny and phylogeny of tooth attachment modes in actinopterygian fishes. J Morphol 167:167-184. 
$\rightarrow$ Fukae M., T. Tanabe, and M. Yamada. 1994. Action of metalloproteinases on porcine dentin mineralization. Calcif Tissue Int 55:426-435.

$\rightarrow$ Gao H., B. Ji, I.L. Jäger, E. Arzt, and P. Fratzl. 2003. Materials become insensitive to flaws at nanoscale: lessons from nature. Proc Natl Acad Sci USA 100:5597-5600.

$\rightarrow$ Geerinckx T, J. De Poorter, and D. Adriaens. 2007. Morphology and development of teeth and epidermal brushes in loricariid catfishes. J Morphol 268:805-814.

$\rightarrow$ Hess W.C. and C. Lee. 1952. The isolation of chondroitin sulfuric acid from dentin. J Dent Res 31:793-797.

Huysseune A. and J.-Y. Sire. 1998. Evolution of patterns and processes in teeth and tooth-related tissues in non-mammalian vertebrates. Eur J Oral Sci 106:437-481.

$\rightarrow$ Ji B. and H. Gao. 2004. Mechanical properties of nanostructure of biological materials. J. Mech. Phys. Solids 52:1963-1990.

$\rightarrow$ Kinney J.H., J.A. Pople, G.W. Marshall, and S.J. Marshall. 2001. Collagen orientation and crystallite size in human dentin: a small angle $x$-ray scattering study. Calcif Tissue Int 69:3137.

$\rightarrow$ Leblond C.P., L.F. Bélanger, and R.C. Greulich. 1955. Formation of bones and teeth as visualized by radioautography. Ann $\mathrm{N}$ Y Acad Sci 60:631-659.

$\rightarrow$ Lormée P., D. Septier, S. Lécolle, C, Baudoin, and M. Goldberg 1996. Dual incorporation of (35S) sulfate into dentin proteoglycans acting as mineralization promotors in rat molars and predentin proteoglycans. Calcif Tissue Int 58:368-375.

Lund R., P. Bartholomew, and A. Kemp. 1992. The composition of the dental hard tissues of fishes. Pp. 35-72 in P. Smith and E. Tchernov, eds. Structure, function and evolution of teeth. Freund, London.

$\rightarrow$ Mannocci F., P. Pilecki, E. Bertelli, and T.F. Watson. 2004. Density of dentinal tubules affects the tensile strength of root dentin. Dent Mater 20:293-296.

$\rightarrow$ Marshall G.W., S.J. Marshall, J.H. Kinney, and M. Balooch $\rightarrow$ 1997. The dentin substrate: structure and properties related to bonding. J Dent 25:441-458.

$\rightarrow$ Miguez P.A., P.N.R. Pereira, P. Atsawasuwan, and M. Yamauchi. 2004. Collagen cross-linking and ultimate tensile strength in dentin. J Dent Res 83:807-810.

$\rightarrow$ Motta P.J. 1984. Tooth attachment, replacement and growth ir $\rightarrow$ Z the butterflyfish, Chaetodon miliaris (Chaetodontidae, Perciformes). Can J Zool 62:183-189.

$\rightarrow$ Nalla R.K., J.H. Kinney, and R.O. Ritchie. 2003. On the fracture of human dentin: is it stress- or strain-controlled? J Biomed Mater Res A 67:484-495.

Nelson J.A., D.A. Wubah, M.E. Whitmer, E.A. Johnson, and D.J. Stewart. 1999. Wood-eating catfishes of the genus $\mathrm{Pa}$ naque: gut microflora and cellulolytic enzyme activities. J Fish Biol 54:1069-1082.

$\rightarrow$ Purcell S.W. and D.R. Bellwood. 1993. A functional analysis of food procurement in two surgeonfish species, Acanthurus nigrofuscus and Ctenochaetus striatus (Acanthuridae). Environ Biol Fish 37:139-159.

$\rightarrow$ Ramsay J.B. and C.D. Wilga. 2007. Morphology and mechanics of the teeth and jaws of white-spotted bamboo sharks (Chiloscyllium plagiosum). J Morphol 268:664-682.

Sreenath T., T. Thyagarajan, B. Hall, G. Longenecker, R. D'Souza, S. Hong, J.T. Wright, M. MacDougall, J. Sauk, and A.B. Kulkarni. 2003. J Biol Chem 278:24874-24880.

$\rightarrow$ Tsuchiya M., Y. Sasano, M. Kagayama, and M. Watanabe. 2001. Characterization of interglobular dentin and Tomes' granular layer in dog dentin using electron probe microanalysis in comparison with predentin. Calcif Tissue Int 68:172-178.

$\rightarrow$ Vlassenbroeck J., M. Dierick, B. Masschaele, V. Cnudde, L. Van Hoorebeke, and P. Jacobs. 2007. Software tools for quantification of x-ray microtomography at the UGCT. Nucl Inst Meth Phys Res A 580:442-445.

Watanabe L.G., G.W. Marshall Jr., and S.J. Marshall. 1996. Dentin shear strength: effects of tubule orientation and intratooth location. Dent Mater 12:109-115.

Waters N.E. 1980. Some mechanical and physical properties of teeth. Pp. 99-135 in J.F.V. Vincent and J.D. Currey, eds. The mechanical properties of biological materials. Cambridge University Press, Cambridge.

$\rightarrow$ Wiesmann H.-P., H.-J. Hohling, K. Zierold, and R. Barckhaust. 1995. Elemental distributions in predentin associated with dentin mineralization in rat incisor. Connect Tissue Res 33: 179-184.

Ye L., M. MacDougall, S. Zhang, Y. Xie, J. Zhang, Z. Li, Y. Lu, Y. Mishina, and J.Q. Feng. 2004. Deletion of dentin matrix protein-1 leads to a partial failure of maturation of predentin into dentin, hypomineralization, and expanded cavities of pulp and root canal during postnatal tooth development. J Biol Chem 279:19141-19148.

Zhang C.Z., H. Li, P.M. Bartold, W.G. Young, and M.J. Waters. 1995. Effect of growth hormone on the distribution of decorin and biglycan during odontogenesis in the rat incisor. J Dent Res 74:1636-1643. 